\title{
APLICAÇÃO DE POLIELETRÓLITO CATIÔNICO PARA DESAGUAMENTO DE LAMA VERMELHA: PRODUÇÃO DE SEDIMENTOS ADENSADOS
}

Bruna Camara Trampus ' Silvia Cristina Alves França '

\section{Resumo}

A indústria mineral gera grandes quantidades de rejeito úmido, e devido aos diversos acidentes que vêm ocorrendo com as barragens convencionais, estudos buscam a máxima eficiência no desaguamento dos mesmos antes de sua disposição. Neste trabalho foram estudadas as propriedades da lama vermelha, a fim de determinar seu comportamento reológico e estabilidade para disposição em barragens de rejeitos. Para tanto, a lama vermelha foi floculada com polímero catiônico comercial e espessada. O sedimento foi submetido a ensaios reológicos com a geometria vane, além do slump test, obtendo informações sobre tensão limite de escoamento e percentagem de abatimento do rejeito adensado. Os resultados mostram grande influência da concentração inicial de sólidos na agregação das partículas, sendo que quanto maior a concentração inicial da polpa, maior a coesão e a tensão limite de escoamento do sedimento, e menor o percentual de abatimento. Foi possível produzir rejeitos adensados, com $\tau_{0}=160 \mathrm{~Pa}$, próximo do especificado para pastas minerais, além de recuperação de cerca de $90 \%$ da água de processo.

Palavras-chave: Reologia; Lama vermelha; Pasta mineral; Tensão de cisalhamento.

\section{APPLICATION OF CATIONIC POLYELECTROLYTE FOR DEWATERING OF THE BAUXITE RESIDUE: PRODUCTION OF THICKENED TAILINGS}

\begin{abstract}
The mineral industry generates large amounts of tailings, and because of many accidents that have occurred with conventional dams, studies focus on tailings dewatering before their disposal. This study evaluates the properties of the red mud to determine their rheological behavior and stability for disposal in dams. For this, the red mud was flocculated with commercial cationic polymer and thickened. The thickened tailing was submitted to rheological tests with the vane rotor and slump test, in order to obtain information about yield stress and slump height of the tailings. The results show a strong influence of the initial solids concentration in particle aggregation, which could be noticed by the increase in particle cohesion and yield stress of thickened tailings; besides reduction of the slump height with the increase of initial solids concentration. It was possible to produce thickened tailings, reaching $\tau_{0}=160 \mathrm{~Pa}$, very close to the value specified for mineral paste, besides up to $90 \%$ water recover.
\end{abstract}

Keywords: Rheology; Red mud; Mineral paste; Yield stress.

\section{INTRODUÇÃO}

Os rejeitos minerais são convencionalmente armazenados em barragens, na forma de polpas adensadas. No entanto, a possibilidade de disposição dos rejeitos na forma de pastas promoverá o reaproveitamento de água de processo - reduzindo a demanda por água nova nas usinas de beneficiamento mineral, além de redução no volume de rejeito e na área necessária para sua disposição, e aumento na estabilidade dos mesmos, reduzindo os riscos de acidentes envolvendo ruptura de barragens e seus impactos negativos ao meio ambiente [I].

Os rejeitos em polpa e pastosos apresentam comportamentos distintos. A polpa é caracterizada por ser um fluido newtoniano, heterogêneo, com baixa concentração de sólidos; seu bombeamento para as barragens é feito por meio de bombas centrífugas, sob regime turbulento, e a segregação e sedimentação das partículas, promove

'Coordenação de Processos Minerais, Centro de Tecnologia Mineral - CETEM, Rio de Janeiro, RJ, Brasil. E-mail: btrampus@cetem.gov.br 
liberação de elevada quantidade de água sobrenadante. Já as pastas minerais são definidas como um material adensado e homogêneo, com baixo ou nenhum nível de água livre; devido à elevada viscosidade, apresentam escoamento laminar, sem segregação granulométrica das partículas, necessitando de bombas de deslocamento positivo. Quando dispostas de forma suave, não apresentam drenagem significativa de água e mínima permeabilidade [1-3].

Conforme as polpas são adensadas para produção das pastas, as suspensões minerais passam a se comportar como fluido não newtoniano com tensão limite de escoamento, que é a tensão mínima necessária para que ocorra a deformação irreversível do material. Tendo as pastas minerais a característica de apresentar tensão limite de escoamento mínima de $200 \mathrm{~Pa}$ [4], esta variável denota a transição entre o comportamento de polpas e pastas. Assim sendo, o monitoramento desse parâmetro é fundamental para o projeto de disposição dos rejeitos como pastas minerais [ $1-4]$.

Inúmeros fatores físico-químicos podem influenciar a floculação e o desaguamento de polpas minerais, como, densidade de cargas superficiais, $\mathrm{pH}$, concentração de sólidos e distribuição granulométrica, sendo a presença de material fino indispensável para a formação das pastas $[3,5,6]$.

Nesse contexto, este estudo busca avaliar o desempenho de um polímero comercial na floculação/espessamento da lama vermelha, resíduo da produção de alumina $\left(\mathrm{Al}_{2} \mathrm{O}_{3}\right)$, visando sua deposição de forma mais adensada, preferencialmente como pastas minerais. Concomitantemente, a qualidade do líquido sobrenadante também foi avaliada, a fim de verificar sua possível reutilização no processo produtivo, ou descarte no meio ambiente, dentro dos parâmetros estabelecidos pela Resolução CONAMA 357/2005 [7].

\section{MATERIAIS E MÉTODOS}

Polpas preparadas com concentração inicial de sólidos $\left(\mathrm{C}_{0}\right)$ variando de 10 a $30 \%(\mathrm{p} / \mathrm{p})$ foram submetidas a ensaios de floculação/sedimentação com um polímero catiônico comercial, nas dosagens (D-pol) de 80 a $200 \mathrm{~g} / \mathrm{t}$, a fim de avaliar a influência da $C_{0}$ e D-pol no adensamento do sedimento. Para tanto, foi realizado um plano experimental fatorial completo a cinco níveis com tréplica no ponto central, totalizando 27 experimentos, como pode ser observado na Tabela I. Além disso, foram realizados ensaios de sedimentação sem a adição de floculante, pra cada $\mathrm{C}_{0}$ estudada.

Tabela I. Plano experimental fatorial completo a cinco níveis e duas variáveis

\begin{tabular}{lccccc}
\hline & $-\mathbf{2}$ & $-\mathbf{I}$ & $\mathbf{0}$ & $\mathbf{+ 1}$ & $\mathbf{+ 2}$ \\
\hline \% sólidos na polpa $(\mathrm{p} / \mathrm{p})-\mathrm{C}_{0}$ & 10 & 15 & 20 & 25 & 30 \\
Dosagem de polímero $(\mathrm{g} / \mathrm{t})-\mathrm{D}$-pol & 80 & 110 & 140 & 170 & 200 \\
\hline
\end{tabular}

\section{I Material}

O rejeito mineral utilizado foi uma amostra de lama vermelha, proveniente de uma refinaria no estado do Pará. A amostra foi seca e desagregada em peneira de $0,42 \mathrm{~mm}$ (35 \#) para preparo da pilha de homogeneização longitudinal e posterior quarteamento manual segmentando a pilha para retirada de alíquotas de $2 \mathrm{~kg}$ e $100 \mathrm{~g}$. No preparo das polpas utilizou-se água da torneira (para simular o uso de água industrial), e como floculante, o polímero catiônico Magnafloc LT7990 ${ }^{\circledR}$, fabricado pela Basf.

\subsection{Caracterização dos Materiais}

A amostra de lama vermelha foi caracterizada em termos de $\mathrm{pH}$ e distribuição granulométrica, em Phmetro Digimed DM-22 e por espalhamento de luz no equipamento Mastersize 2000 - Malvern, respectivamente. O rejeito mineral e o polímero floculante foram caracterizados com relação a densidade de carga superficial no equipamento Zetasizer nano series - Malvern, na faixa de $\mathrm{pH}$ de 2 a I2, ajustado com soluções diluídas de $\mathrm{KOH}$ e $\mathrm{HCl}$, e em eletrólito indiferente, $\mathrm{KCl}(0,0 \mathrm{I}$ e $0,00 \mathrm{I} \mathrm{M})$.

\subsection{Caracterização do Rejeito Adensado}

As polpas foram preparadas e floculadas em equipamento Jar Test, no qual o polímero foi adicionado à polpa sob rotação de $300 \mathrm{rpm}$, mantendo-se a agitação durante Imin; em seguida, a velocidade de agitação foi reduzida para $150 \mathrm{rpm}$, agitando-se por mais $2 \mathrm{~min}$; e, posteriormente transferiu-se a polpa floculada para uma proveta de $2 \mathrm{~L}$ (Figura I), deixando em repouso para sedimentação. A turbidez do líquido clarificado (sobrenadante) foi analisada após I e $2 \mathrm{~h}$ de sedimentação, em turbidímetro portátil da Hach, modelo 2 I O0P. O material permaneceu em repouso por $24 \mathrm{~h}$, e após esse período, o líquido sobrenadante foi removido e o sedimento de lama vermelha estudado segundo as propriedades, equipamentos e técnicas descritos nos itens a seguir.

\subsection{Teste de abatimento - slump test}

O teste de abatimento permite determinar a consistência do rejeito mineral, sendo de grande importância para as operações de disposição dos rejeitos [I]. O ensaio é padronizado para geometria cônica, e convencionalmente

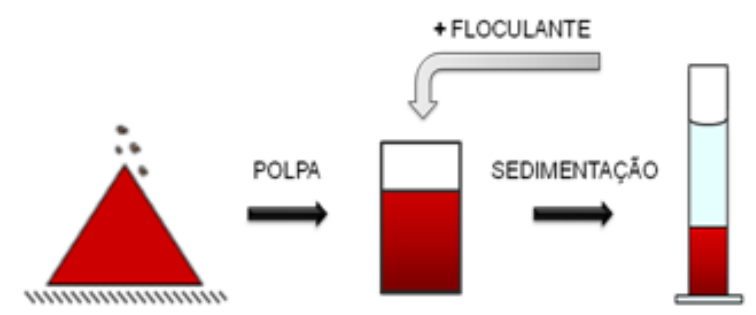

Figura I. Esquema referente às etapas do procedimento experimental utilizado. 
utilizado para determinar a consistência do concreto [8]. No entanto, trabalhos relatam que é possível obter resultados bem similares adaptando a metodologia para utilização de cilindros em PVC, com altura igual ao diâmetro interno, sendo este o procedimento adotado nesse estudo [9].

O procedimento experimental pode ser observado no esquema da Figura 2, e consiste em preencher o cilindro com o sedimento do fundo da proveta, removê-lo verticalmente, medir, em quatro pontos, a altura do sedimento, e calcular a diferença entre a altura do cilindro $(\mathrm{H})$ e altura média do sedimento $\left(\mathrm{H}_{\mathrm{s}}\right)$ (Equação I). Nesse estudo, utilizou-se um cilindro com $\mathrm{H}=\mathrm{D}_{\text {in }}=0,053 \mathrm{~m}$, e o \% ABT é calculado de acordo com a Equação 2.

$$
\mathrm{h}=\mathrm{H}-\mathrm{H}_{\mathrm{s}}
$$

$$
\% \mathrm{ABT}=(\mathrm{h} / \mathrm{H}) \times 100
$$

\subsubsection{Análise reológica}

A tensão limite de escoamento $\left(\tau_{0}\right)$ foi determinada para o topo e o fundo do sedimento contido na proveta, utilizando-se o reômetro e o rotor vane. A metodologia experimental foi baseada em diferentes estudos da litertura $[I-3,10,1 \mathrm{I}]$, os quais relatam que a utilização do rotor vane para determinação do $\tau_{0}$ impondo-se uma baixa

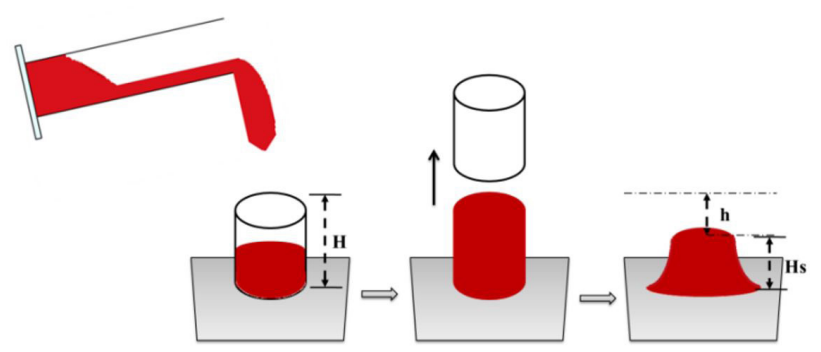

Figura 2. Esquema do teste de abatimento com cilindro.

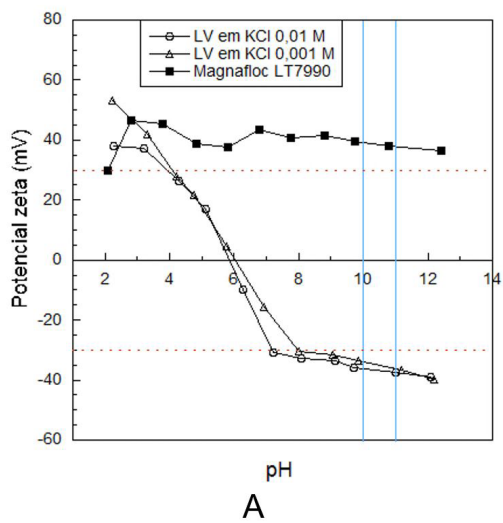

rotação constante, é bem similar ao obtido pela curva de fluxo, e é aplicável às suspensões minerais. Assim sendo, o procedimento experimental foi realizado em três etapas à temperatura ambiente $\left(25^{\circ} \mathrm{C}\right)$. Primeiramente, a tensão limite de escoamento do sedimento estruturado foi analisada aplicando uma deformação constante de I rpm por $180 \mathrm{~s}$, sendo referida como $\tau_{0}$ estática. Após isso, o sedimento foi submetido a uma taxa de cisalhamento constante de $100 \mathrm{~s}^{-1}$ por um período de $180 \mathrm{~s}$, a fim de destruir a estrutura dos flocos e liberar a água contida. Esse sedimento tem seu ponto de fluidez referido como $\tau_{0}$ dinâmica, e foi analisado por duas formas distintas: a primeira igual a anterior, ou seja, mantendo uma rotação constante de $1 \mathrm{rpm}$ durante $180 \mathrm{~s}$, e posterior a isso, realizou-se uma curva de fluxo variando a taxa de cisalhamento $(\gamma)$ de $100 \mathrm{~s}^{-1}$ a $0,01 \mathrm{I} \mathrm{s}^{-1}$, com 10 pontos por década, e cada ponto obtido em um intervalo máximo de $5 \mathrm{~min}$. A tensão limite de escoamento foi determinada pela curva de fluxo aplicando-se o modelo de Herschel-Bulkley, descrito na Equação 3.

$$
\tau=\tau_{0}+K \dot{\gamma}^{n}
$$

Sendo: $\mathrm{K}=$ índice de consistência do fluido; $\mathrm{n}$ = índice relacionado ao comportamento do fluido.

\subsubsection{Determinação da concentração do sedimento}

Após os ensaios reológicos, as amostras foram transferidas para a balança de umidade Ohaus modelo MB23, e submetidas a aquecimento para a determinação da concentração do sedimento.

\section{RESULTADOS E DISCUSSÃO}

\section{I Caracterização da Amostra de Lama Vermelha}

O rejeito mineral estudado é caracterizado por uma granulometria muito fina, $\mathrm{d}(0,9)=16,22 \mu \mathrm{m} ; \mathrm{d}(0,5)=4,32 \mu \mathrm{m}$; $\mathrm{d}(0, \mathrm{I})=\mathrm{I}, \mathrm{I} 4 \mu \mathrm{m}, 90 \%$ de suas partículas têm diâmetro inferior a $16,22 \mu \mathrm{m}$. A associação dessa característica à forte repulsão eletrostática existente em $\mathrm{pH}$ natural $(10 \leq \mathrm{pH} \leq \mathrm{I})$,

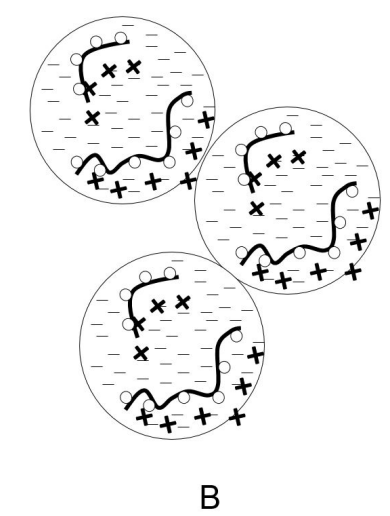

Figura 3. (A) Análise da densidade de carga superficial dos materiais utilizados; (B) Formação de flocos pelo mecanismo de neutralização das cargas. 
devido a densidade de cargas aniônicas (Figura 3A), dificulta o processo de sedimentação natural e, consequentemente, a formação dos sedimentos adensados e a recuperação de água [3]. Na mineração, polímeros são amplamente utilizados como floculantes de polpas, e suas características mais influentes para a eficiência de formação dos flocos são a massa molecular e a densidade de cargas [5]. O floculante utilizado, Magnafloc LT 7990, é um polieletrólito catiônico (Figura 3A) de massa molecular média, e conforme pode ser observado na Figura 3A, sua densidade de cargas é similar a da lama em pH natural, porém com carga oposta. Devido à sua elevada densidade de cargas opostas a das partículas em suspensão, sua adsorção na superfície dessas partículas ocorre pelo mecanismo de atração eletrostática com neutralização das cargas e consequente redução das forças repulsivas [3,5, 12]. Assim, formam-se flocos (Figura 3B) facilitando o processo de sedimentação, e, aumentando a eficiência da separação sólido-líquido. Em consequência a similaridade da densidade de cargas, é provável alcançar a neutralização das cargas com menores dosagens do polímero, minimizando os custos.

\subsection{Caracterização do Rejeito Adensado}

Os rejeitos produzidos com base nos experimentos apresentados na Tabela I apresentaram concentração de sólidos $\left(\mathrm{C}_{\text {sed }}\right)$ na faixa de $5 \mathrm{I}$ a $62 \%(\mathrm{p} / \mathrm{p})$, \% ABT entre 57 e $86 \%$, $\tau_{0}$ estático de 65 a $163 \mathrm{~Pa}$ e $\tau_{0}$ dinâmico de 12 a $40 \mathrm{~Pa}$, para o fundo do sedimento.

Com relação aos ensaios reológicos, a Figura 4 A exibe os valores de tensão para o topo e fundo do sedimento da polpa com $C_{0}=30 \%$, floculada com l $40 \mathrm{~g} / \mathrm{t}$ de Magnafloc. Observa-se que ambas as metodologias aplicadas para determinação da $\tau_{0}$ dos sedimentos foram concordantes, ou seja, o $\tau_{0}$ determinado, impondo-se uma deformação constante, é bem similar ao obtido pela curva de fluxo e o modelo Herschel-Bulkley, conforme relatado por Boger [I].

Para todos os casos estudados, segundo o planejamento experimental (Tabela I), o topo do sedimento apresentou $\mathrm{C}_{\text {sed }}$ de 15 a $25 \%$ menor que a do fundo, apresentando maiores valores de $\tau_{0}$ estática e dinâmica (Figura 4A). Observa-se, também, a variação da $\tau_{0}$ do sedimento em função do perfil de concentração existente, sendo possível obter os extremos de $\tau_{0}$ em cada caso analisado. A Figura $4 \mathrm{~B}$ exibe os resultados da triplicata no ponto central do planejamento, permitindo a observação de elevada reprodutibilidade nos resultados. Assim, pôde-se associar pequenos erros aos dados de $\tau_{0}$ estático e dinâmico.

A Figura $5 \mathrm{~A}$ mostra a dependência da $\tau_{0}$ com a dosagem de Magnafloc para as polpas com $30 \%$ de sólidos. É possível observar que a adição de floculante à polpa gera elevação da $\tau_{0}$ estática, porém o aumento da dosagem de Magnafloc não altera os valores de $\tau_{0}$ - estática e dinâmica - do sedimento, sendo estas função de $\mathrm{C}_{0}$ (Figura 6B). O mesmo comportamento foi observado para todas as variações de $\mathrm{C}_{0}$, podendo-se inferir que a adição do Magnafloc permitiu, inicialmente, maior adensamento do sedimento, pois agregou as partículas sólidas gerando maior acúmulo de água no interior do floco, e consequentemente, a elevação da $\tau_{0}$ estática. Assim, conclui-se que $80 \mathrm{~g} / \mathrm{t}$ do polieletrólito catiônico são suficientes para alcançar o máximo de neutralização das cargas e formação dos flocos, possibilitando avaliar dosagens menores, que acarretará na redução de custos.

A análise da $\tau_{0}$ do sedimento floculado após cisalhamento $\left(100 \mathrm{~s}^{-1}\right), \tau_{0}$ dinâmica, tem o intuito de simular o máximo de destruição dos flocos devido ao bombeamento do rejeito, do sedimentador até o local de deposição. Porém, visto que para as pastas a bomba centrífuga é substituída pela de deslocamento positivo, a intensidade de destruição poderá ser menor, devendo ser analisada futuramente.

A Figura 6 mostra os valores obtidos de \% ABT, em função das variáveis estudadas. Analisando-se a Figura 6A, pode-se observar que a adição de floculante à polpa produz sedimentos mais consistentes, o que é evidenciado pela redução do \% $A B T$, em comparação com a sedimentação da polpa natural. A maior eficiência no adensamento dos sedimentos foi alcançada para as polpas com $\mathrm{C}_{0}$ a partir de $20 \%(\mathrm{p} / \mathrm{p})$. Observa-se também que para polpas

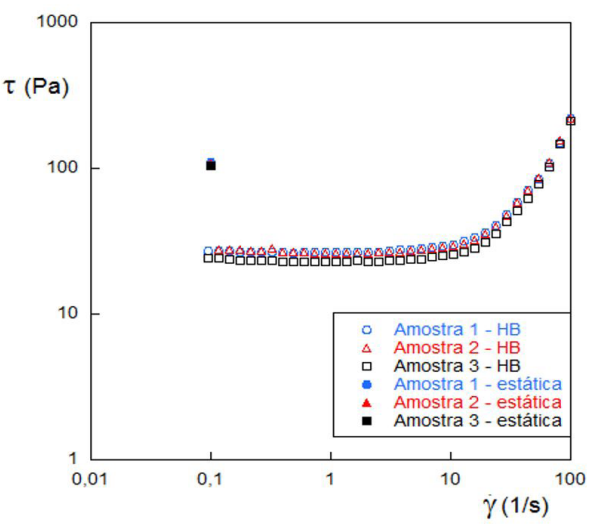

B

Figura 4. Curva de escoamento para o sedimento de lama vermelha. (A) $C_{0}=30 \%, D$-pol $=140 \mathrm{~g} / \mathrm{t}$, $C_{\text {sed topo }}=42,8 \%, C_{\text {sed fundo }}=56,6 \%$; (B) fundo do sedimento no ponto central do planejamento experimental, $C_{0}=20 \%, D-p o l=140 \mathrm{~g} / \mathrm{t}, C_{\text {sed } 1}=56,6 \%, C_{\operatorname{sed} 2}=58,4 \%, C_{\text {sed } 3}=56,7 \%$. 


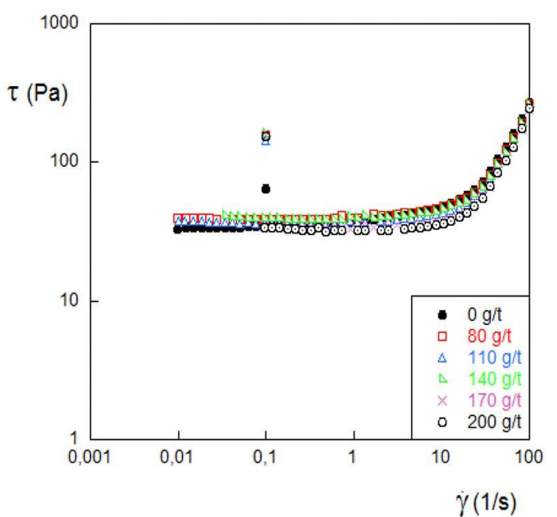

A

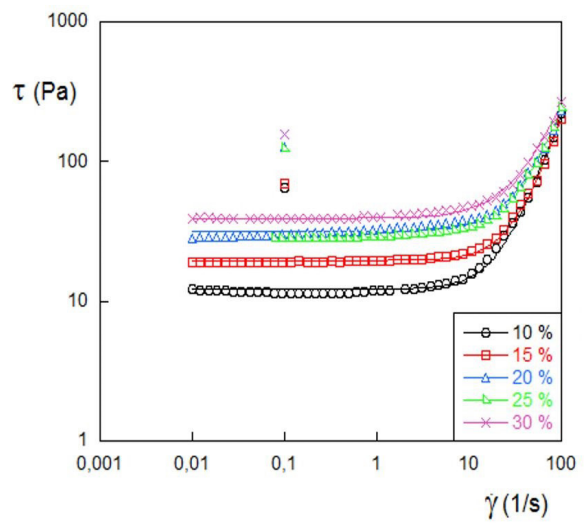

B

Figura 5. Curvas de escoamento para o sedimento de lama vermelha. $(A) C_{0}=30 \%$, variando $D$-pol; (B) 80 g/t de Magnafloc, variando $C_{0}$.

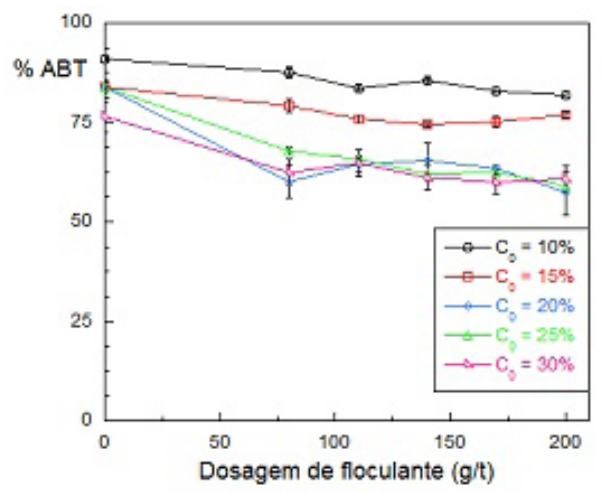

A

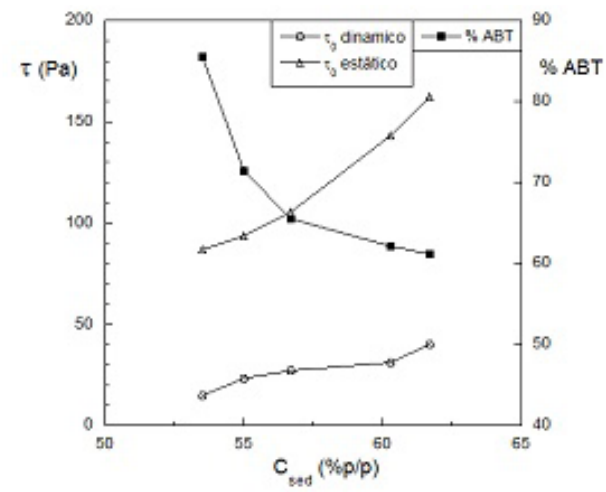

B

Figura 6. Gráficos de correlação das variáveis estudadas para o fundo do sedimento floculado. (A) \% ABT e D-pol, variando $C_{0}$; $(B) \tau_{0}$, $C_{\text {sed’ }}$, $\% A B T$ para as polpas floculadas com I $40 \mathrm{~g} / \mathrm{t}$ de Magnafloc.

com mesmo $\mathrm{C}_{0}$, o aumento de $\mathrm{D}$-pol não influencia, de maneira significativa, o empacotamento das partículas, pois considerando os erros associados, observ a-se que os dados de \% ABT são constantes, corroborando com os resultados obtidos de $\tau_{0}$ - estática e dinâmica.

A Figura 6B, exibe a correlação entre o\% $\mathrm{ABT}, \mathrm{C}_{\text {sed }}$ e $\tau_{0}$ para as polpas floculadas com $140 \mathrm{~g} / \mathrm{t}$ de Magnafloc. Nota-se que para sedimentos mais adensados, maior $\mathrm{C}_{\text {sed }}$, tem-se maior $\tau_{0}$ e a consequente redução do \% ABT. O \% ABT é uma medida empírica da consistência do rejeito, e, conforme estudado por Clayton et al. [9] tem relação inversa com a $\tau_{0}$ e com a densidade do material. Estudos [I-3, I0] relatam que a tensão limite de escoamento cresce exponencialmente com a concentração de sólidos, ou seja, um pequeno aumento da concentração de sólidos no sedimento, leva ao crescimento da $\tau_{0}$, e consequentemente, a redução do $\% A B T$. No presente estudo, tal fenômeno pode ser observado na Figura 6B, pois o crescimento de $C_{0}$, implica em sedimentos mais adensados e com menores \% ABT. Resultados semelhantes foram obtidos por Hernandez et al. [6] para polpas de rejeito de cobre do Chile, e por Boger [I] para diferentes amostra de lama vermelha.
A Figura 7 expõe a interferência do Magnafloc no adensamento do sedimento; pode-se observar que apesar do processo de sedimentação da polpa natural produzir sedimentos com $\mathrm{C}_{\text {sed }}$ ligeiramente mais elevados, os sedimentos gerados com o uso do floculante são mais coesos, possuindo maiores valores de $\tau_{0}$ estática - em alguns casos apresentam aumento superior a $100 \%$ (Figura 7 ) - e menor \% ABT.

\subsection{Análise da Viabilização do Reuso da Água}

A água é um dos principais insumos da indústria mineral, tornando-se essencial viabilizar o seu reaproveitamento. Os resultados de turbidez do líquido clarificado após $2 \mathrm{~h}$ de sedimentação são apresentados na Figura 8A. Pode-se observar que a utilização do floculante Magnafloc, resultou na expressiva redução de turbidez do líquido clarificado, alcançando até $97 \%$ de redução, em comparação com a sedimentação natural. Assim, pode-se observar que a floculação com um polímero catiônico permitiu, de forma eficiente, a neutralização das cargas e consequente desestabilização e sedimentação das partículas finas que permaneciam em suspensão. O Magnafloc, em todos os casos estudados, promoveu a redução da turbidez do sobrenadante para 


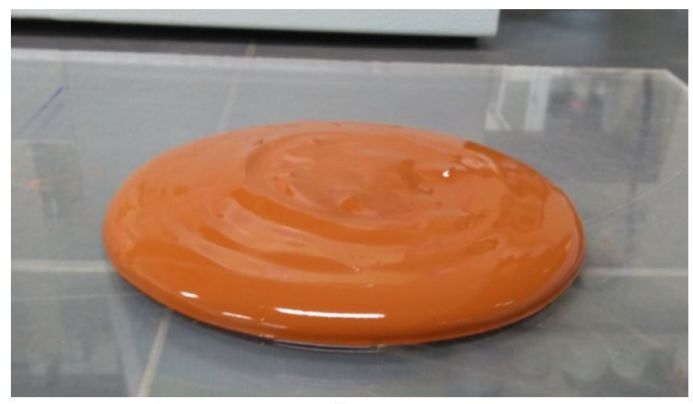

A

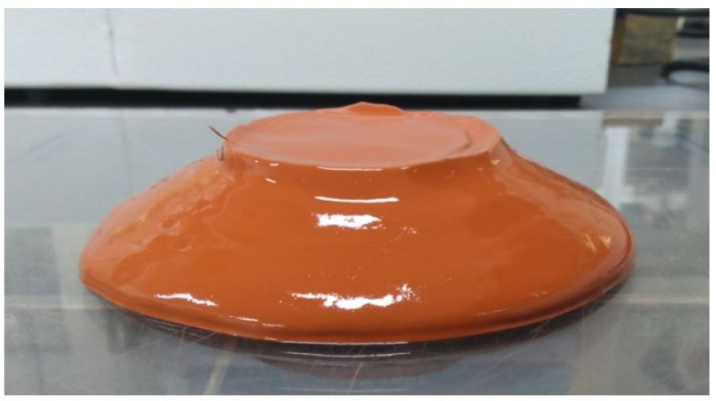

B

Figura 7. Teste de abatimento. (A) $C_{0}=30 \%$ sem floculante, $C_{\text {sed }}=63 \%, \tau_{0}$ estática $=64 \mathrm{~Pa}$ e $\% \mathrm{ABT}=77 ;(\mathrm{B}) \mathrm{C}_{0}=30 \%, \mathrm{D}$-pol $=80 \mathrm{~g} / \mathrm{t}$, $\mathrm{C}_{\text {sed }}=61 \%, \tau_{0}$ estática $=157 \mathrm{~Pa}$ e $\% \mathrm{ABT}=63$.

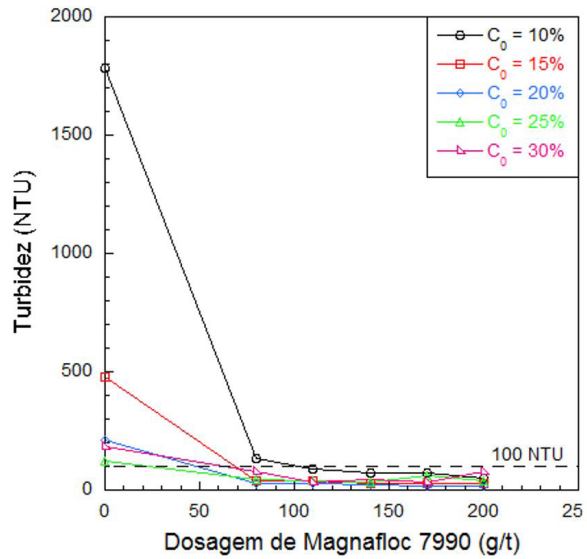

A

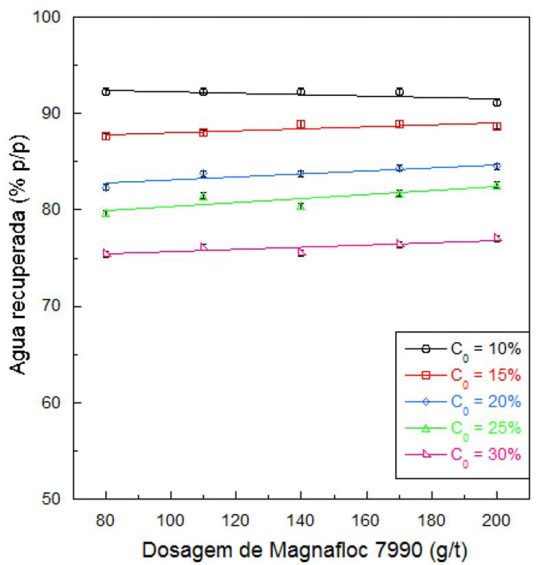

B

Figura 8. Água recuperada (\% p/p) em função da concentração de sólidos e dosagem de floculante.

valores abaixo de $100 \mathrm{NTU}$, que era o desejado, visto que está dentro dos parâmetros estabelecidos pela Resolução CONAMA 357/2005 [7], e viabiliza o reuso da água no processo, ou até seu descarte no meio ambiente.

O gráfico da Figura 8B mostra que a floculação da lama vermelha com Magnafloc, seguida de espessamento descontínuo, permite recuperação de água em até $92 \%$. Observa-se também que o aumento de $D$-pol não apresenta grande interferência no desaguamento, provavelmente pode estar ocasionando até uma inversão de cargas na solução mantendo a repulsão de algumas partículas finas. Contudo, estudos mais aprofundados com análises de potencial zeta são necessários para fornecer uma melhor explicação. Com relação a $C_{0}$, é nítida a forte dependência da recuperação de água com $C_{0}$. Sendo que, o aumento de $C_{0}$ gera redução no percentual de água recuperado, pois ocorre a formação de maior quantidade de flocos, e consequentemente, maior aprisionamento de água.

\section{CONCLUSÕES}

Analisando a eficiência de aplicação do polieletrólito catiônico como floculante frente às variáveis estudadas dentro do planejamento experimental aplicado, pode-se concluir que o aumento da concentração inicial de sólidos na polpa, promove o expressivo crescimento do adensamento do sedimento, exibindo maior coesão e $\tau_{0}$, e menor \% ABT. Este processo se mostrou eficiente para desaguar o rejeito, proporcionando sedimentos com $\tau_{0}$ estático $=160 \mathrm{~Pa}$, próximo ao característico de pastas, e reduzidos $\% A B T$, para a menor dosagem de floculante estudada $(80 \mathrm{~g} / \mathrm{t})$.

Com foco na gestão sustentável do recurso hídrico, pode-se afirmar que a aplicação do polieletrólito catiônico é viável para reduzir a água livre presente nas barragens convencionais, além de permitir o reuso da água de processo. Para ensaios com $2 \mathrm{~h}$ de sedimentação e a menor dosagem de Magnafloc estudada $(80 \mathrm{~g} / \mathrm{t})$, é possível obter sobrenadante com valores de turbidez abaixo de 100 NTU, que é um dos parâmetros estabelecidos pela Resolução CONAMA $357 / 2005$ [7] para o reuso da água.

Vale resaltar que o aumento da dosagem do polímero não modificou as características do sedimento frente à sua coesão/adensamento, e com relação à turbidez do sobrenadante, a redução foi pouco expressiva. Assim, a menor dosagem estudada $(80 \mathrm{~g} / \mathrm{t})$ já foi suficiente para alcançar as características desejadas. Contudo, pode-se ainda avaliar/estudar a eficiência de menores dosagens do Magnafloc, visando menor custo. 
O estudo da agregação/sedimentação da lama vermelha foi mais eficiente utilizando-se o polímero Magnafloc LT7990 como floculante, em comparação às polpas sem tratamento, pois permitiu a formação de sedimentos mais adensados, e a consequente redução da água livre das barragens convencionais.

Diante do exposto, os resultados oferecem às refinarias de alumina uma alternativa para o gerenciamento/disposição da lama vermelha, visando processos mais sustentáveis com a reutilização da água. $A$ continuidade do trabalho pode convergir para redução da dosagem de polímero, a fim de avaliar o enquadramento do sobrenadante aos parâmetros estabelecidos pela Resolução CONAMA 357/2005 [7] para reuso ou descarte.

\section{Agradecimentos}

Ao CNPq, pelo apoio financeiro (CTUniversal Proc. $421710 / 2016-0)$ e à empresa BASF pela amostra de floculante.

\section{REFERÊNCIAS}

I Boger DV. Rheology of slurries and environmental impacts in the mining industry. Annual Review of Chemical and Biomolecular Engineering. 2013;4:239-257.

2 Sofrá F, Boger DV. Environmental rheology for waste minimisation in the minerals industry. Chemical Engineering Journal. 2002;86:319-330.

3 Sofrá F, Boger DV. Rheology for thickened tailings and paste - history, state-of-the-art and future directions. In: Australian Centre for Geomechanics, The University of Western Australia. Proceedings of the 14th International Seminar on Paste and Thickened Tailings; 5-7 April 20II; Perth, Australia. Australia: The University of Western Australia; 20II. p. I2I-I33.

4 Fitton TG, Seddon KD. Paste and thickened tailings - myth busting and clarification. In: Proceedings of the 16th International Seminar on Paste and Thickened Tailings; 2013 June 17-20; Belo Horizonte, Brazil. Australia: The University of Western Australia; 2013. p. 37-43.

5 Gregory J, Barany S. Adsorption and flocculation by polymers and polymer mixtures. Advances in Colloid and Interface Science. 201 I; 169:I-12.

6 Hernandez CA, Quiero AL, Alegria PI. Efeito da distribuição granulométrica sobre o comportamento reológico de pastas minerais. In: Universidade Federal de Alfenas. Anais do $26^{\circ}$ Encontro Nacional de Tratamento de Minérios e Metalurgia Extrativa; 2015 Outubro I8-22; Poços de Caldas, Brazil. Alfenas: Universidade Federal de Alfenas; 20 I5. P. 421-429.

7 Brasil. Conselho Nacional do Meio Ambiente. Resolução CONAMA n 357, de 17 de março de 2005. Dispõe sobre a classificação dos corpos de água e diretrizes ambientais para o seu enquadramento, bem como estabelece as condições e padrões de lançamento de efluentes, e dá outras providências. Diário Oficial da União. 2005 Mar 18.

8 American Society for Testing and Materials. Annual Book of ASTM Standards. Designation: CI43/C I43M-97 Standard Test Method for Slump of Hydraulic-Cement Concrete. Vol. 04.02 - Concrete and Aggregates. West Conshohocken: ASTM International; 1998. p. 89.

9 Clayton S, Grice TG, Boger DV. Analysis of the slump test for on-site yield stress measurement of mineral suspensions. International Journal of Mineral Processing. 2003;70:3-2.

10 Nguyen QD, Boger DV. Application of rheology to solving tailings disposal problems. International Journal of Mineral Processing. 1998;54:217-233.

I I Nguyen QD, Boger DV. Yield stress measurement for concentrated suspensions. Journal of Rheology (New York, N.Y.). 1983;27(4):32I-349.

12 Campêlo LD, Baltar CAM, França SCA. The importance of an initial aggregation step for the destabilization of an anatase colloidal suspension. Colloids and Surfaces. A, Physicochemical and Engineering Aspects. 2017;531:67-72.

Recebido em: 26 Out. 2018

Aceito em: 20 Ago. 2019 